\title{
Simulation of 2D Elastic Bodies with Randomly Distributed Circular Inclusions Using the BEM
}

\author{
Zhenhan Yao ${ }^{1}$, Fanzhong Kong ${ }^{2}$, Xiaoping Zheng ${ }^{1}$ \\ ${ }^{1}$ Department of Engineering Mechanics \\ ${ }^{2}$ State Key Lab of Automotive Safety and Energy \\ Tsinghua University \\ Beijing, 100084 China
}

\begin{abstract}
Based on the Rizzo's direct boundary integral equation formulation for elasticity problems, elastic bodies with randomly distributed circular inclusions are simulated using the boundary element method. The given numerical examples show that the boundary element method is more accurate and more efficient than the finite element method for such type of problems. The presented approach can be successfully applied to estimate the equivalent elastic properties of many composite materials.
\end{abstract}

\section{Introduction}

As composite materials are applied more and more to many important engineering projects, researchers have paid much more attention to the simulation of the composite materials and to the estimation of their equivalent elastic properties.

To estimate the equivalent elastic properties of composite materials, many theoretical models have been developed, such as the composite cylinder model ${ }^{[1]}$, the dilute or non-interacting solution ${ }^{[2]}$, the self-consistent method ${ }^{[3]}$, the generalized self-consistent method $^{[4 \sim 7]}$ and the Mori-Tanaka method ${ }^{[8 \sim 11]}$. At the same time, only few data obtained from numerical simulations can be found in literature. A numerical procedure, based on the series expansion of complex potentials, was proposed in Ref. ${ }^{[12]}$, but only several kinds of periodic arrays of holes were considered. A spring force model has been used to simulate a sheet containing circular holes arranged as triangular and hexagonal $\operatorname{arrays}^{[13]}$. A numerical equivalent inclusion method was presented ${ }^{[14]}$, which can be applied to analyze stress fields in and around inclusions of various shapes by the finite element method. But this approach can only be applied to simulate the elastic body with only one inclusion. The simulation of the sheet with randomly or normally distributed circular holes was investigated in our group several years ago ${ }^{[15]}$.

Based on Rizzo's direct BEM ${ }^{[16]}$ for elasticity problem, a new BEM approach for the simulation of elastic bodies with randomly distributed circular inclusions is proposed in this paper. Several numerical examples are presented to menstruate the advantages of this new BEM approach over the domain-based FEM approach. 


\section{BEM for simulation of 2D elastic body with a circular inclusion}

The model of $2 \mathrm{D}$ elastic body with a circular inclusion is shown in Figure 1, where $\Omega_{\mathrm{I}}$, $\Omega_{\mathrm{II}}$ denote the domain of matrix and inclusion respectively, $\Gamma^{i}$ the matrix-inclusion interface boundary, and $\Gamma_{\mathrm{I}}^{t}, \Gamma_{\mathrm{I}}^{u}$ indicate the given traction part and given displacement part of the outer boundary of matrix material domain $\Gamma_{\mathrm{I}}$.

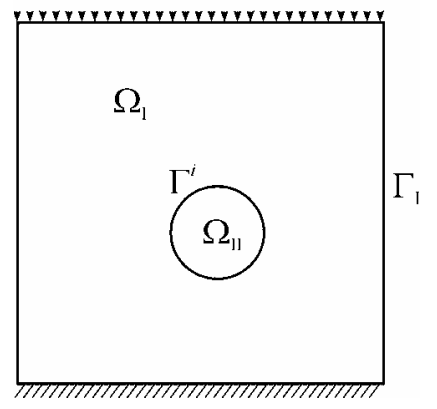

Fig. 1. Model of 2D elastic body with a circular inclusion

The boundary integral equations can be written for the matrix and inclusion subdomain respectively:

$$
\begin{aligned}
& C_{\alpha \beta}(p) u_{\beta}(p)=\int_{\Gamma_{\Gamma}+\Gamma^{i}} U_{\alpha \beta}(p, q) t_{\beta}^{\mathrm{I}}(q) \mathrm{d} \Gamma-\int_{\Gamma_{1}+\Gamma^{i}} T_{\alpha \beta}^{\mathrm{I}}(p, q) u_{\beta}(q) \mathrm{d} \Gamma \\
& C_{\alpha \beta}(p) u_{\beta}(p)=\int_{\Gamma^{i}} U_{\alpha \beta}(p, q) t_{\beta}^{\mathrm{II}}(q) \mathrm{d} \Gamma-\int_{\Gamma^{i}} T_{\alpha \beta}^{\mathrm{II}}(p, q) u_{\beta}(q) \mathrm{d} \Gamma
\end{aligned}
$$

Where superscripts I and II indicate matrix and inclusion subdomain respectively, $p$ and $q$ stand for the source point of fundamental solution and field point on the boundary respectively, $C_{\alpha \beta}(p)$ is a free term determined from the shape of the boundary at source point $p, U_{\alpha \beta}(p, q), T_{\alpha \beta}^{\mathrm{I}}(p, q)$ and $T_{\alpha \beta}^{\mathrm{II}}(p, q)$ are fundamental solutions of $2 \mathrm{D}$ elasticity problem, and $u_{\beta}, t_{\beta}$ are boundary displacement and boundary traction respectively.

After discretization using linear or quadric boundary elements, equations (1) can be rewritten into matrix form as follows:

$$
\begin{aligned}
& {\left[\begin{array}{lll}
\boldsymbol{A}_{11}^{\mathrm{I}} & \boldsymbol{A}_{12}^{\mathrm{I}} & \boldsymbol{A}_{13}^{\mathrm{I}} \\
\boldsymbol{A}_{21}^{\mathrm{I}} & \boldsymbol{A}_{22}^{\mathrm{I}} & \boldsymbol{A}_{23}^{\mathrm{I}} \\
\boldsymbol{A}_{31}^{\mathrm{I}} & \boldsymbol{A}_{32}^{\mathrm{I}} & \boldsymbol{A}_{33}^{\mathrm{I}}
\end{array}\right]\left\{\begin{array}{l}
\boldsymbol{U}^{\mathrm{I}} \\
\boldsymbol{T}^{\mathrm{I}} \\
\boldsymbol{U}^{i}
\end{array}\right\}=\left[\begin{array}{lll}
\boldsymbol{B}_{11}^{\mathrm{I}} & \boldsymbol{B}_{12}^{\mathrm{I}} & \boldsymbol{B}_{13}^{\mathrm{I}} \\
\boldsymbol{B}_{21}^{\mathrm{I}} & \boldsymbol{B}_{22}^{\mathrm{I}} & \boldsymbol{B}_{23}^{\mathrm{I}} \\
\boldsymbol{B}_{31}^{\mathrm{I}} & \boldsymbol{B}_{32}^{\mathrm{I}} & \boldsymbol{B}_{33}^{\mathrm{I}}
\end{array}\right]\left\{\begin{array}{l}
\overline{\boldsymbol{T}}^{\mathrm{I}} \\
\overline{\boldsymbol{U}}^{\mathrm{I}} \\
\boldsymbol{T}^{i \mathrm{I}}
\end{array}\right\}} \\
& \boldsymbol{G}^{\mathrm{II}} \boldsymbol{T}^{\mathrm{iII}}=\boldsymbol{H}^{\mathrm{II}} \boldsymbol{U}^{i}
\end{aligned}
$$


where $\boldsymbol{U}^{\mathrm{I}}$ and $\overline{\boldsymbol{T}}^{\mathrm{I}}$ represent the unknown nodal displacement vector and the given nodal traction vector respectively on the given traction boundary $\Gamma_{\mathrm{I}}^{t}, \boldsymbol{T}^{\mathrm{I}}$ and $\overline{\boldsymbol{U}}^{\mathrm{I}}$, the unknown nodal traction vector and the given nodal displacement vector on the given displacement boundary $\Gamma_{\mathrm{I}}^{u}, \boldsymbol{U}^{i}$, the unknown nodal displacement vector on the matrix-inclusion interface boundary $\Gamma^{i}$. While $\boldsymbol{T}^{i \mathrm{I}}$ and $\boldsymbol{T}^{i \mathrm{II}}$ stand for the unknown nodal traction vector on the matrix-inclusion interface boundary for the matrix and inclusion material respectively.

For the matrix material, the subdomain is a multiply connected domain, the nodal number and the corresponding sequence of boundary variables can be arranged sequentially in the positive direction of the boundary. When advancing in the positive direction of boundary, the inner domain surrounded by the boundary is always at the left side. For the inclusion, the nodal number of the matrix-inclusion interface should keep in line with that of matrix material subdomain. So the nodal number and the corresponding sequence of boundary variables should be arranged sequentially in the negative direction of the boundary for the inclusion itself.

In equations (2), the displacement continuity on the matrix-inclusion interface has been taken into account. The interface condition for the tractions can be written as:

$$
\boldsymbol{T}^{i \mathrm{II}}=-\boldsymbol{T}^{i \mathrm{I}}
$$

Substituting equation (3) into the equation (2b), we can obtain the relation between the tractions and displacements of the matrix on the interface:

$$
\boldsymbol{T}^{i \mathrm{I}}=-\left(\boldsymbol{G}^{\mathrm{II}}\right)^{-1} \boldsymbol{H}^{\mathrm{II}} \boldsymbol{U}^{i}
$$

Substituting equation (4) into equation (2a), we can obtain the final system of equations for the 2D elastic body with a circular inclusion as follows:

$$
\left[\begin{array}{ccc}
\boldsymbol{A}_{11}^{\mathrm{I}} & \boldsymbol{A}_{12}^{\mathrm{I}} & \boldsymbol{A}_{13}^{\mathrm{I}}+\boldsymbol{B}_{13}^{\mathrm{I}}\left(\boldsymbol{G}^{\mathrm{II}}\right)^{-1} \boldsymbol{H}^{\mathrm{II}} \\
\boldsymbol{A}_{21}^{\mathrm{I}} & \boldsymbol{A}_{22}^{\mathrm{I}} & \boldsymbol{A}_{23}^{\mathrm{I}}+\boldsymbol{B}_{23}^{\mathrm{I}}\left(\boldsymbol{G}^{\mathrm{II}}\right)^{-1} \boldsymbol{H}^{\mathrm{II}} \\
\boldsymbol{A}_{31}^{\mathrm{I}} & \boldsymbol{A}_{32}^{\mathrm{I}} & \boldsymbol{A}_{33}^{\mathrm{I}}+\boldsymbol{B}_{33}^{\mathrm{I}}\left(\boldsymbol{G}^{\mathrm{II}}\right)^{-1} \boldsymbol{H}^{\mathrm{II}}
\end{array}\right]\left\{\begin{array}{l}
\boldsymbol{U}^{\mathrm{I}} \\
\boldsymbol{T}^{\mathrm{I}} \\
\boldsymbol{U}^{i}
\end{array}\right\}=\left[\begin{array}{ll}
\boldsymbol{B}_{11}^{\mathrm{I}} & \boldsymbol{B}_{12}^{\mathrm{I}} \\
\boldsymbol{B}_{21}^{\mathrm{I}} & \boldsymbol{B}_{22}^{\mathrm{I}} \\
\boldsymbol{B}_{31}^{\mathrm{I}} & \boldsymbol{B}_{32}^{\mathrm{I}}
\end{array}\right]\left\{\begin{array}{l}
\overline{\boldsymbol{T}}^{\mathrm{I}} \\
\overline{\boldsymbol{U}}^{\mathrm{I}}
\end{array}\right\}
$$

\section{BEM for simulation of 2D elastic body with randomly distributed circular inclusions}

The model of $2 \mathrm{D}$ elastic body with randomly distributed identical circular inclusions is shown in Figure 2. Where $\Omega_{0}$ is the subdomain of matrix material, $\Omega_{1}, \Omega_{2}, \cdots, \Omega_{i}$, $\cdots, \Omega_{n}$, the subdomains of inclusion material, $\Gamma_{i}$ the matrix-inclusion interface boundaries, and $\Gamma_{0}$ the outer boundary of the matrix material subdomain. 


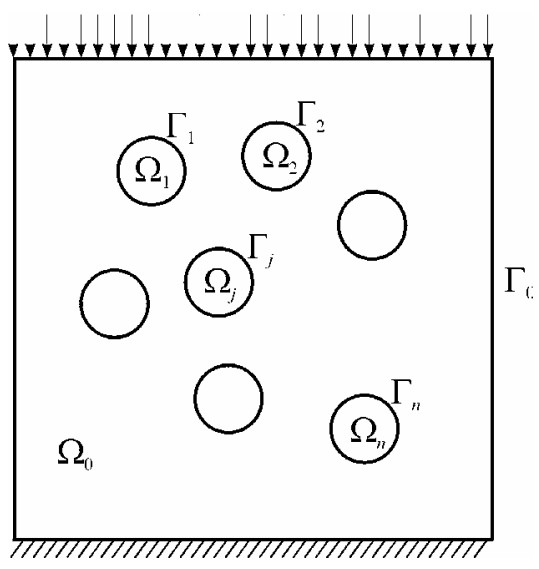

Fig. 2. Model of 2D elastic body with randomly distributed identical circular inclusions

If the conventional subdomain boundary element method is adopted, $n+1$ equation systems for the $n+1$ subdomains should be solved. As the number of inclusions increases, the computing time will increase significantly. If we notice that the relations between the tractions and displacements of each identical inclusion are just the same and similar as shown in equation (4), we can reduce the full computation to the solution of the equation for the matrix material domain with inner boundary conditions similar to equation (4), which can be described as follows:

$$
\left[\begin{array}{ccccccc}
\boldsymbol{A}_{11} & \boldsymbol{A}_{12} & \tilde{\boldsymbol{A}}_{13}^{1} & \cdots & \tilde{\boldsymbol{A}}_{13}^{i} & \cdots & \tilde{\boldsymbol{A}}_{13}^{n} \\
\boldsymbol{A}_{21} & \boldsymbol{A}_{22} & \tilde{\boldsymbol{A}}_{23}^{1} & \cdots & \tilde{\boldsymbol{A}}_{23}^{i} & \cdots & \tilde{\boldsymbol{A}}_{23}^{n} \\
\boldsymbol{A}_{31}^{1} & \boldsymbol{A}_{32}^{1} & \tilde{\boldsymbol{A}}_{33}^{11} & \cdots & \tilde{\boldsymbol{A}}_{33}^{1 i} & \cdots & \tilde{\boldsymbol{A}}_{33}^{n} \\
\vdots & \vdots & \vdots & \ddots & \vdots & \ddots & \vdots \\
\boldsymbol{A}_{31}^{i} & \boldsymbol{A}_{32}^{i} & \tilde{\boldsymbol{A}}_{33}^{i 1} & \cdots & \tilde{\boldsymbol{A}}_{33}^{i i} & \cdots & \tilde{\boldsymbol{A}}_{33}^{i n} \\
\vdots & \vdots & \vdots & \ddots & \vdots & \ddots & \vdots \\
\boldsymbol{A}_{31}^{n} & \boldsymbol{A}_{32}^{n} & \tilde{\boldsymbol{A}}_{33}^{n 1} & \cdots & \tilde{\boldsymbol{A}}_{33}^{n i} & \cdots & \tilde{\boldsymbol{A}}_{33}^{n n}
\end{array}\right]\left\{\begin{array}{c}
\boldsymbol{U} \\
\boldsymbol{T} \\
\boldsymbol{U}^{1} \\
\vdots \\
\boldsymbol{U}^{i} \\
\vdots \\
\boldsymbol{U}^{n}
\end{array}\right\}=\left[\begin{array}{cc}
\boldsymbol{B}_{11} & \boldsymbol{B}_{12} \\
\boldsymbol{B}_{21} & \boldsymbol{B}_{22} \\
\boldsymbol{B}_{31}^{1} & \boldsymbol{B}_{32}^{1} \\
\vdots & \vdots \\
\boldsymbol{B}_{31}^{i} & \boldsymbol{B}_{32}^{i} \\
\vdots & \vdots \\
\boldsymbol{B}_{31}^{n} & \boldsymbol{B}_{32}^{n}
\end{array}\right]\left\{\begin{array}{l}
\boldsymbol{T} \\
\overline{\boldsymbol{U}}
\end{array}\right\}
$$

where

$$
\begin{aligned}
& \tilde{\boldsymbol{A}}_{13}^{i}=\boldsymbol{A}_{13}^{i}+\boldsymbol{B}_{13}^{i}(\boldsymbol{G})^{-1} \boldsymbol{H} \\
& \tilde{\boldsymbol{A}}_{23}^{i}=\boldsymbol{A}_{23}^{i}+\boldsymbol{B}_{23}^{i}(\boldsymbol{G})^{-1} \boldsymbol{H} \\
& \tilde{\boldsymbol{A}}_{33}^{i j}=\boldsymbol{A}_{33}^{i j}+\boldsymbol{B}_{33}^{i j}(\boldsymbol{G})^{-1} \boldsymbol{H}
\end{aligned}
$$

The first and the second subscript indicate the boundary where the source point node $p$ and the field point node $q$ located, and 1,2, 3 denote the traction given part, displacement given part of outer boundary and the inner boundaries respectively. To distinguish different inner boundaries, the superscripts are used. The first and the second (if there is a second one) superscript indicate the number of inner boundary where the 
source point node $p$ and the field point node $q$ located. Matrices $\boldsymbol{G}$ and $\boldsymbol{H}$ in equation (7) are coefficient matrixes for the inclusion material subdomains. As all the randomly distributed circular inclusions are identical, it needs to form the coefficient matrixes $\boldsymbol{G}$ and $\boldsymbol{H}$ for a certain inclusion only one time. In equation (6), $\boldsymbol{U}, \boldsymbol{T}$ and $\boldsymbol{U}^{i}$ indicate the unknown displacement vector on the traction given part of outer boundary, the unknown traction vector on the displacement given part of outer boundary and the unknown displacement vector on the $i$-th inner interface boundaries respectively. On the other hand, $\overline{\boldsymbol{T}}$ and $\overline{\boldsymbol{U}}$ stand for the given traction vector and the given displacement vector on the outer boundary respectively.

For the case of 2D elastic body with randomly distributed circular inclusions of different size, the above-presented approach can be generalized no difficulty, provided the number of different size is much less than the number of inclusions. In such case, the equation (7) should be modified as follows:

$$
\begin{aligned}
& \tilde{\boldsymbol{A}}_{13}^{i}=\boldsymbol{A}_{13}^{i}+\boldsymbol{B}_{13}^{i}\left(\boldsymbol{G}^{k(i)}\right)^{-1} \boldsymbol{H}^{k(i)} \\
& \tilde{\boldsymbol{A}}_{23}^{i}=\boldsymbol{A}_{23}^{i}+\boldsymbol{B}_{23}^{i}\left(\boldsymbol{G}^{k(i)}\right)^{-1} \boldsymbol{H}^{k(i)} \\
& \tilde{\boldsymbol{A}}_{33}^{i j}=\boldsymbol{A}_{33}^{i j}+\boldsymbol{B}_{33}^{i j}\left(\boldsymbol{G}^{k(i)}\right)^{-1} \boldsymbol{H}^{k(i)}
\end{aligned}
$$

where $k=1,2, \cdots, m$ denote different inclusion size, $k(i)$ can be also a random function, and for the inclusion of each size the matrices $\boldsymbol{G}$ and $\boldsymbol{H}$ should be computed once.

\section{Numerical examples}

1) A square sheet with a circular inclusion at the center subjected to uniform tension on two opposite edges

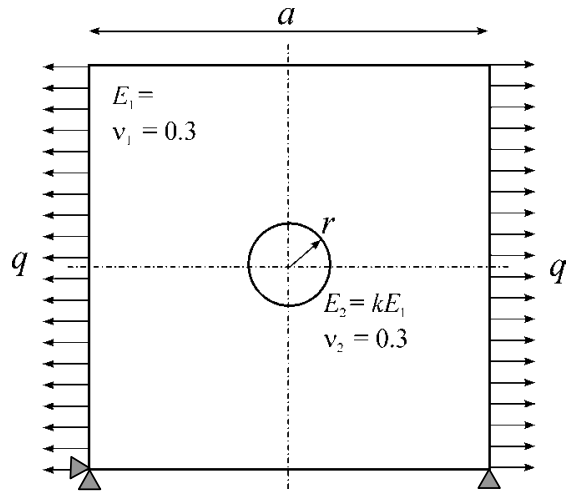

Fig. 3. Model of a square sheet with a circular inclusion at the center subjected to uniform tension on two opposite edges 
Figure 3 shows the computational model. The side length $a=100 \mathrm{~mm}$, the radius of the circular inclusion $r=2 \mathrm{~mm}$, the traction $q=10 \mathrm{MPa}$, the material properties of the matrix $E_{1}=10 \mathrm{MPa}, v_{1}=0.3$, and that of the inclusion $E_{2}=k E_{1}$ and $v_{2}=0.3$. The rigid body displacement is constrained properly. In the computation, the matrix-inclusion interface is divided into 10 quadratic elements.

Figure 4 shows the absolute value of circumferential stress $\left|\sigma_{\theta}\right|$ on the matrixinclusion interface obtained by BEM in comparison with the analytical solution, for the special case of circular hole. The solid line is the analytical solution, and the solid dots show the BEM results. As to the case of an infinite sheet with a circular hole at the center, the analytical solution can be written as:

$$
\left|\sigma_{\theta}\right|=q|1-2 \cos 2 \theta|
$$

It is obvious that the maximum of the circumferential stress $\left|\sigma_{\theta}\right|$ is $3 q=30 \mathrm{MPa}$ when $\theta$ is equal to $\pi / 2$ or $3 \pi / 2$, and the minimum is zero when $\theta$ is equal to $\pi / 6,5 \pi / 6,7 \pi / 6$ or $11 \pi / 6$. It can be found that the present numerical results agree with the analytical solution very well. The maximum error of BEM results is less than $0.02 \%$.

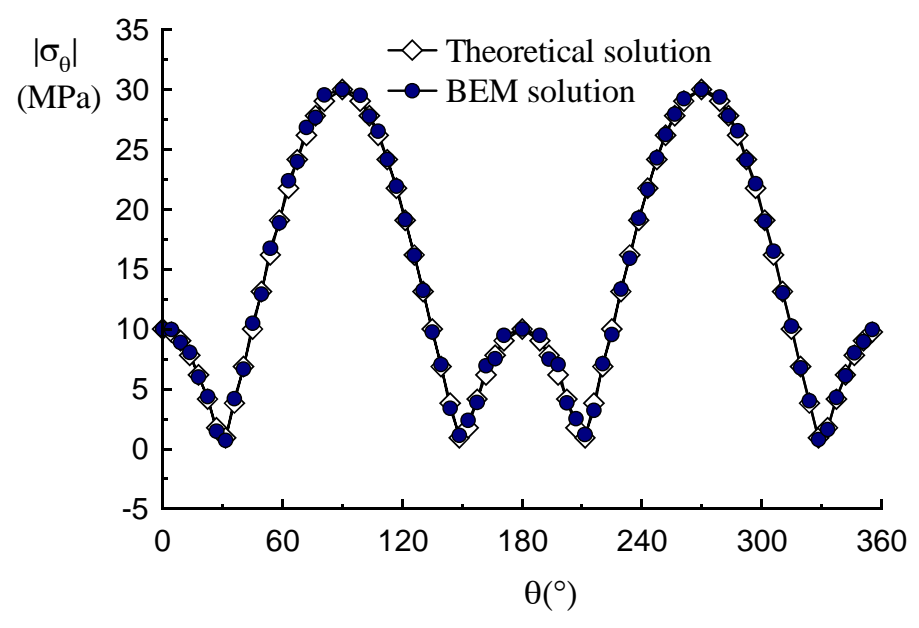

Fig. 4. Comparison of the circumferential stress $\left|\sigma_{\theta}\right|$ on the matrix- inclusion interface obtained by the BEM and the analytical solution 


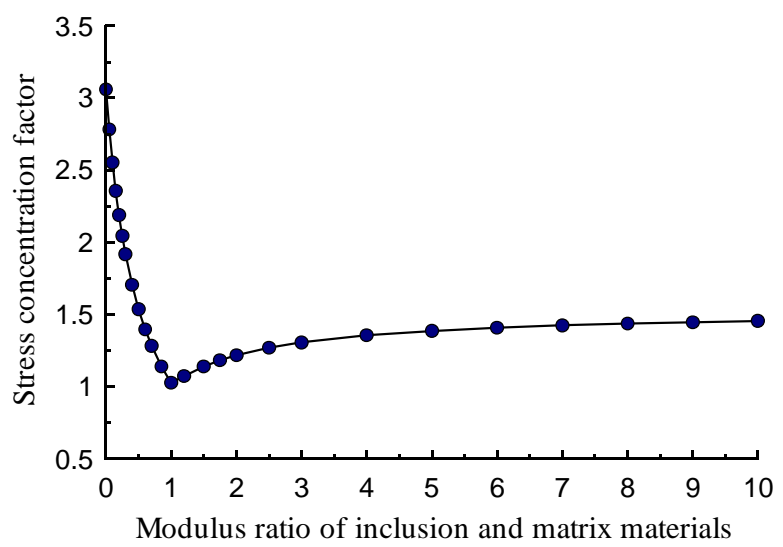

Fig. 5. Relation between stress concentration factor and the inclusion-matrix modulus ratio obtained from BEM scheme

Figure 5 shows that the stress concentration factor obtained by BEM varies with the inclusion-matrix modulus ratio, the solid dots show the numerical results, and the solid line is interpolated curve. It can be found from Figure 5 that the stress concentration factor decreases quickly with the increase of inclusion-matrix modulus ratio when inclusion material is softer than matrix material. Furthermore, stress concentration factor increases slowly with the increase of the inclusion-matrix modulus ratio when inclusion material is harder than matrix material. As to the analytical solution of an infinite sheet with a circular hole or a circular rigid core at the center, the stress concentration factor is equal to 3.0 or 1.51 from elastic theory respectively. The corresponding results obtained by BEM are 3.0005 and 1.51002 respectively. It is obvious that the present numerical results agree with the elastic theory very well.

2) A square sheet with two very close inclusions subjected to uniform displacement on one edge

Figure 6 shows the computational model. The side length $a=100 \mathrm{~mm}$, the radius of two very close circular inclusions $R=5 \mathrm{~mm}$, the minimum distance between the matrix-inclusion interface boundaries $b=0.5 \mathrm{~mm}$, the given uniform displacement on the right edge $d=1.0 \mathrm{~mm}$, the material property of the matrix $E_{1}=10 \mathrm{MPa}, v_{1}=0.3$, and that of the inclusion $E_{2}=k E_{1}, v_{2}=0.3$.

It is obvious that the stress concentration factor increases to the maximum when the inclusion-matrix modulus ratio approaches zero. So the stress gradient around the matrix-inclusion interface will reach the maximum for the case of two circular holes. To ensure high accuracy, it is necessary to take finer mesh around the two inclusions for either the BEM or FEM computation.

Figure 7 shows the variation of Von-Mises stress on one quarter of the matrix-inclusion 
interface by using 10 quadratic boundary elements and 20 quadratic boundary elements. It can be found that the variation is very small, which indicates a convergent solution has been obtained by using only 10 quadratic boundary elements in BEM computation.

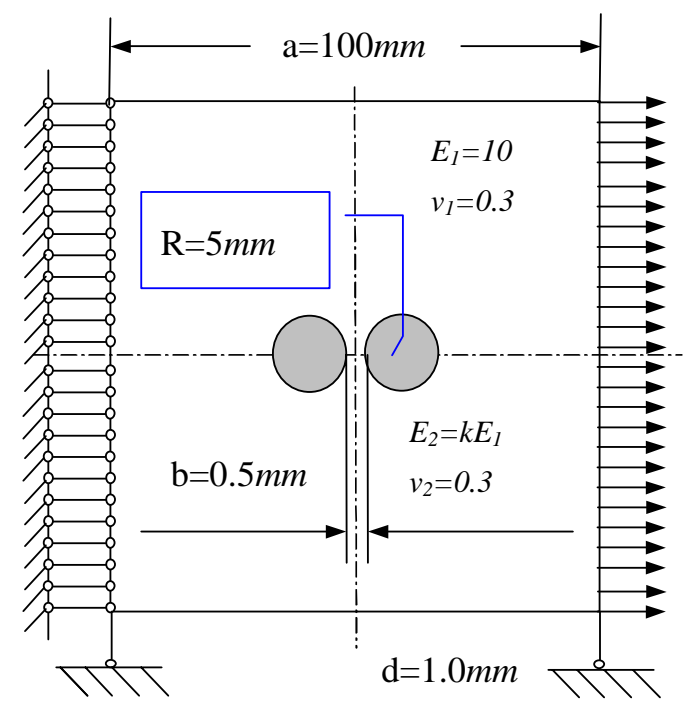

Fig. 6. The model of a square sheet with two very close inclusions under given uniform displacement on one edge

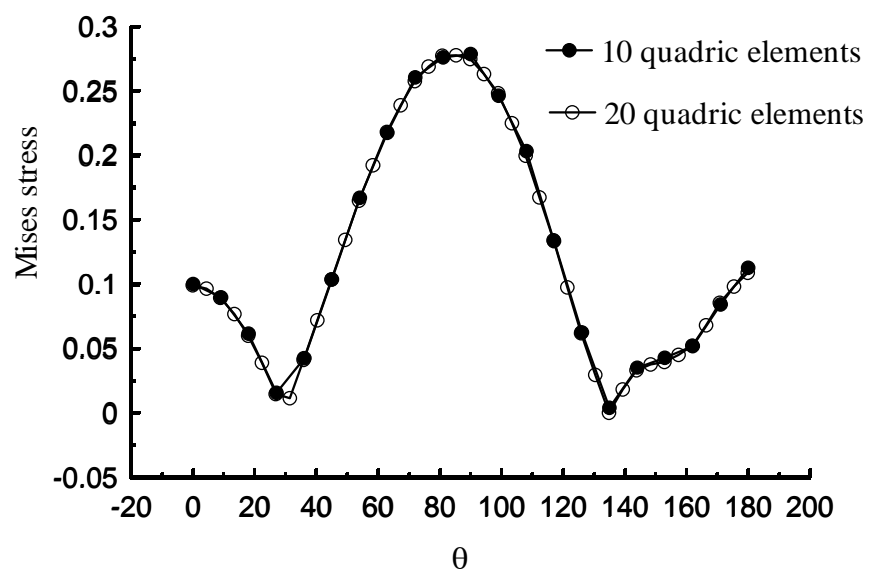

Fig. 7. Comparison of the Von-Mises stress on the quarter of the matrix-inclusion boundaries by different boundary element number 


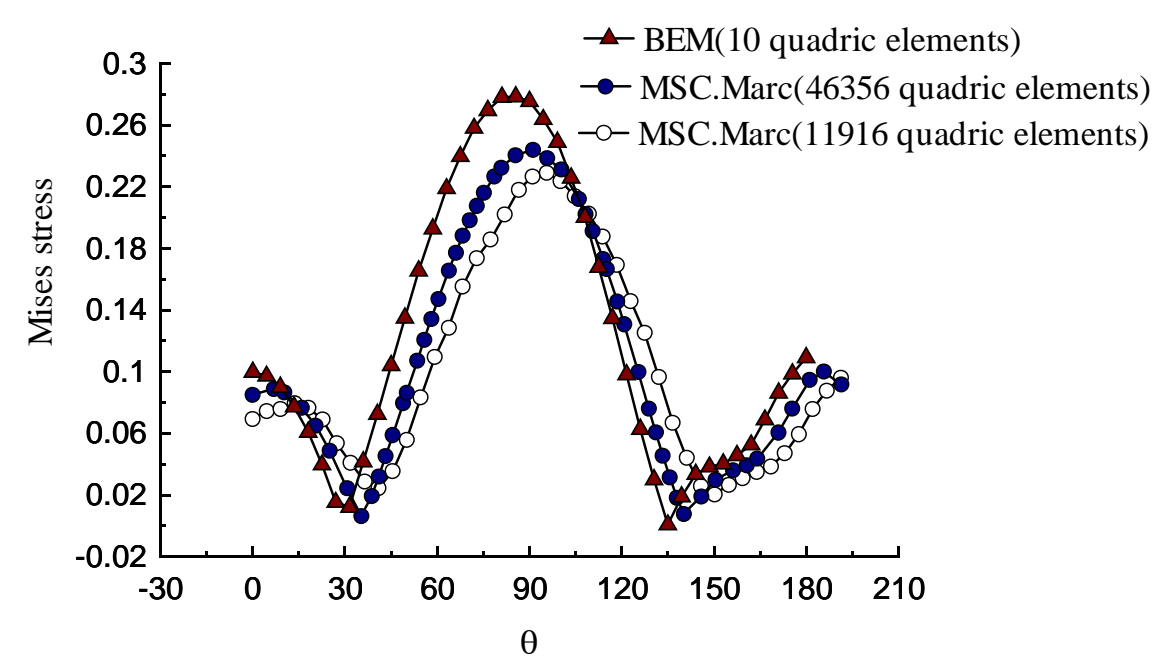

Fig. 8. Comparison of Von-Mises stress on the quarter of the matrix-inclusion interface boundary obtained from BEM and the famous MSC.Marc software

Figure 8 shows the comparison of Von-Mises stress on one quarter of matrix-inclusion interface obtained by the presented BEM and the FEM using MSC/Marc software, for the case of two circular holes. It can be found that the Von-Mises stress increases slowly to the results obtained by BEM with the immense increase of finite elements. It is obvious that the accuracy reached by MSC/Marc using 46356 quadratic elements is far lower than by BEM using 10 quadratic boundary elements on the interface. The presented BEM is much more effective than FEM for such kind of problems. Furthermore, for 2D elastic body with randomly distributed circular inclusions, there will be plenty of very close inclusions. Thus, the presented scheme of BEM is very suitable to such simulation problems, and it has obvious advantage over the FEM.

3) A square sheet with 100 randomly distributed identical circular inclusions subjected to uniform tension on the opposite edges

Figure 9 shows the computational model. The side length of this square sheet is $100 \mathrm{~mm}$, the traction on the opposite edges is $10 \mathrm{Mpa}$, the thickness of this square sheet is $1 \mathrm{~mm}$, and the volume ratio of all 100 inclusions is 0.4 . Then the radius of the circular inclusion can be determined automatically. The model is taken as a plane stress problem. In addition, domain mesh is only needed for plotting results.

After BEM computation, we can obtain the deformation pattern and stress distribution. As examples, Figure 10 shows the deformation pattern for the case of 100 circular holes, $E_{2}=0$, Figure 11 and Figure 12 shows the Von-Mises stress distribution of a square sheet with 100 randomly distributed identical circular hole under the uniform tension on two opposite edges for the case of $E_{2} / E_{1}=0.5$. 


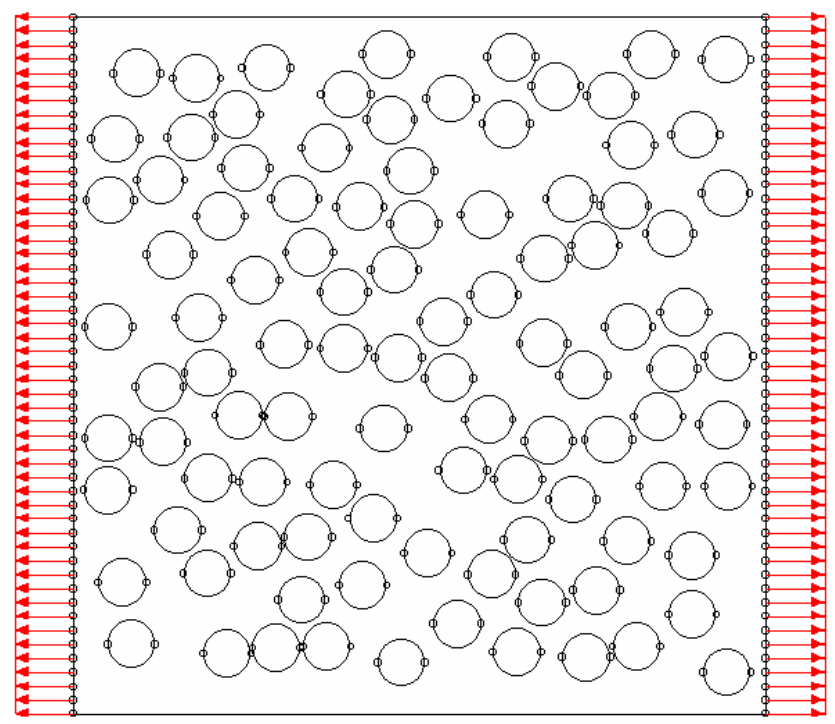

Fig. 9. Model of a square sheet with 100 randomly distributed identical circular inclusions under uniform tension on two opposite edges

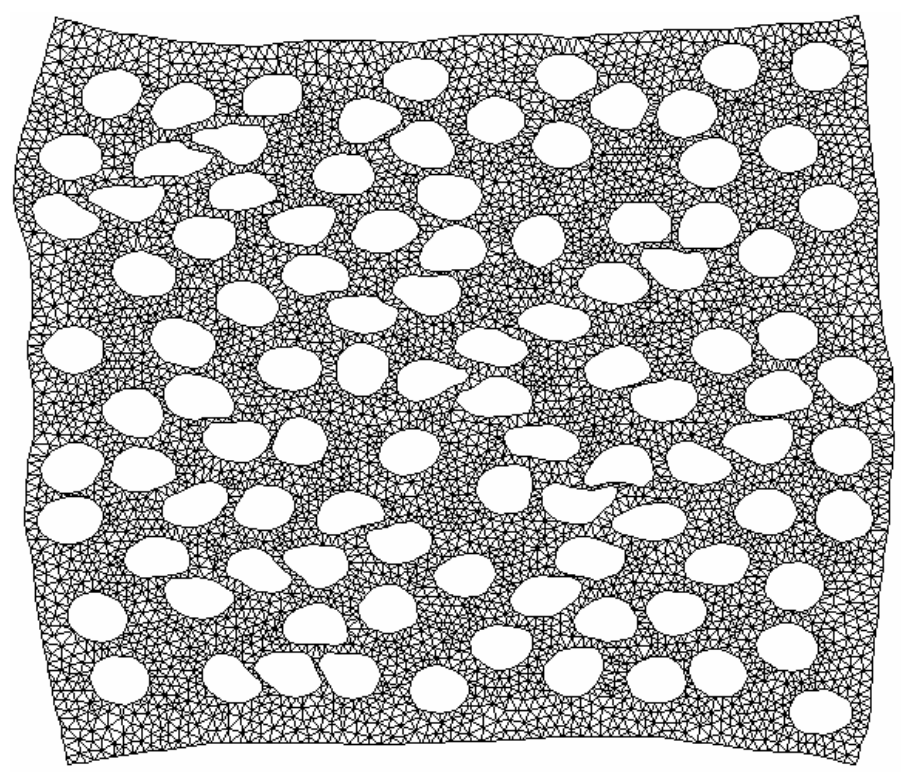

Fig. 10. Deformation pattern of a square sheet with 100 randomly distributed identical circular hole under the uniform tension on two opposite edges $\left(E_{2}=0\right)$ 


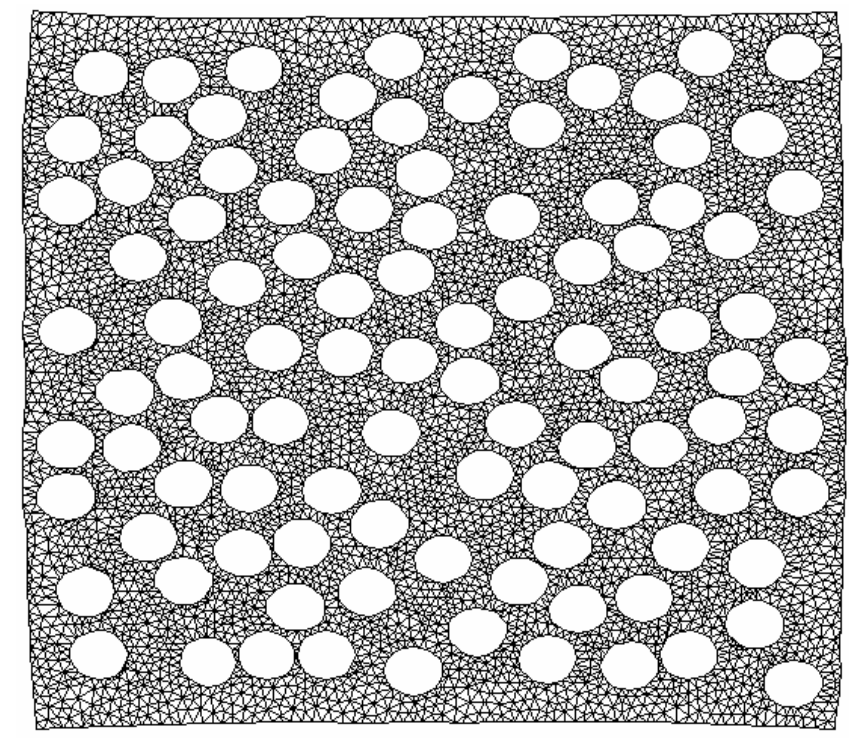

Fig. 11. Deformation pattern of a square sheet with 100 randomly distributed identical circular inclusions under the uniform tension on two opposite edges $\left(E_{2} / E_{1}=0.5\right)$

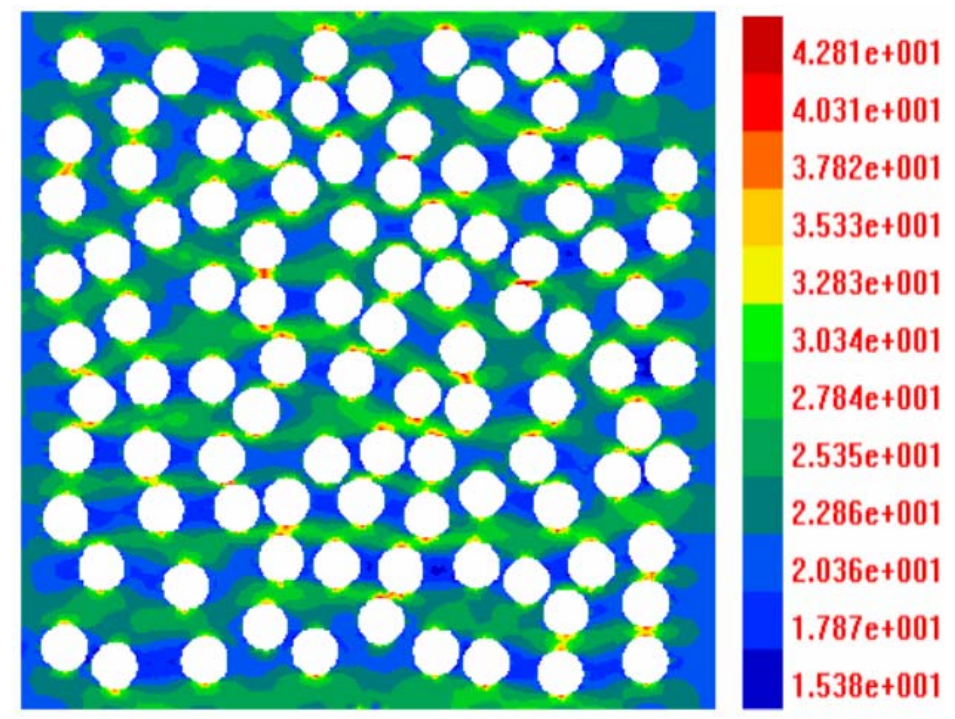

Fig.12. Von-Mises stress distribution of a square sheet with 100 randomly distributed identical circular holes under uniform tension on two opposite edges $\left(E_{2} / E_{1}=0.5\right)$ 


\section{Concluding Remarks}

1) A scheme of the BEM for the simulation of $2 \mathrm{D}$ elastic bodies with randomly distributed circular inclusions has been presented in this paper. The given numerical examples indicate its high accuracy and high efficiency.

2) As for the elastic bodies with randomly distributed identical circular inclusions, the presented BEM scheme has a distinctive advantage over the FEM due to high stress gradient resulted from the presence of many very close inclusions.

3) The presented BEM scheme can be generalized without difficulty to the elastic bodies with randomly distributed inclusions of different geometrical sizes, different shapes (elliptical inclusion with different shape and principal direction, cracks with different direction, etc.) and different elastic modulus.

4) The presented BEM scheme can be applied to estimate the equivalent elastic properties of corresponding composite materials.

5) The presented BEM can be combined with some kind of fast multipole algorithms. It is possible to efficiently simulate the elastic bodies with much more different inclusions with such fast algorithms.

Since this manuscript was submitted two years ago, some related investigations in the authors' group have been published ${ }^{[17-20]}$. In those investigations, a large number of numerical examples of the $2 \mathrm{D}$ elastic solids with randomly distributed inclusions, using repeated similar sub-domain BEM, have shown that this method has higher accuracy and higher efficiency, and provides an efficient tool for the numerical simulations of corresponding composite materials. This method can be applied to simulate not only the 2D solids with inclusions of different shapes, sizes and materials, but also the inclusions with interphases layers. By applying the fast multipole BEM into this field, the scale of the computation can be increased. In a preliminary investigation, the number of inclusions simulated was increased from 100 to 1600.

Further investigations will be carried out in two directions: on the one hand, it will be developed from 2D to 3D problems; on the other hand from the simulation of effective elastic moduli to simulations of the failure process of such composite materials.

\section{Acknowledgements}

Financial support for the project from the National Natural Science Foundation of China under grant No. 19772025 and No. 10172053 is gratefully acknowledged.

\section{References}

[1] Hashin Z. The elastic moduli of heterogeneous materials. J Appl Mech., 1962; 29: 143 150.

[2] Budiansky Y. On the elastic moduli of heterogeneous materials. J Mech Phys Solids, 1965; 13: 223 227. 
[3] Hill R. A self-consistent mechanics of composite materials. J Mech Phys Solids, 1965; 13: 213 222.

[4] Christensen RM, Lo KH. Solutions for effective shear properties in three phase sphere and cylinder models. J Mech Phys Solids, 1979; 27: 315 330.

[5] Aboudi J, Benveniste Y. The effective moduli of cracked bodies in plane deformation. Engng Fracture Mech., 1987; 26: 171 184.

[6] Huang Y, Hu KX, Wei X, Chandra A. A generalized self-consistent mechanics method for composite materials with multiphase inclusions, J Mech Phys Solids, 1994; 42: 491 504.

[7] Huang Y, Hu KX, Chandra A. A generalized self-consistent mechanics method for microcracked solids, J Mech Phys Solids, 1994; 42: 1273 1291.

[8] Mori T, Tanaka K. Average stress in matrix and average elastic energy of materials with mis-fitting inclusions. Acta Metal, 1973; 21: 571 583.

[9] Taya M, Chou T-W. On two kinds of ellipsoidal inhomogeneities in an infinite elastic body: an application to a hybrid composite. Int J Solids Structure, 1979; 27: 315 330.

[10] Weng GJ. Some elastic properties of reinforced solids, with special reference to isotropic ones containing spherical inclusions. Int J Engng Sci., 1984; 22: $845 \sim 856$.

[11] Benveniste Y. A new approach to the application of Mori-Tanaka theory in composite materials. Mech Mater, 1987; 6: 147 157.

[12] Isida M, Igawa H. Analysis of zigzag array of circular holes in an infinite solid under uniaxial tension. Int J Solids Structure, 1991; 27: 849 864.

[13] Day AR, Snyder KA, Garboczi EJ, Thorpe MF. The elastic moduli of a sheet containing circular holes. J Mech Phys Solids 1992; 40: 1031-1051.

[14] Yuji Nakasone, Hirotada Nishiyama, Tetsuharu Nojiri. Numerical equivalent inclusion method: a new computational method for analyzing stress fields in and around inclusions of various shapes, Materials Science and Engineering, 2000; A285: 229 238.

[15] Hu N, Wang B, Tan GW, Yao ZH, Yuan WF. Effective elastic properties of 2-D solids with circular holes: numerical simulations, Composites Science and Technology, 2000; 60:1811-1823.

[16] Rizzo FJ. An integral equation approach to boundary value problems of classical elastostatics. Quart. J. of Appl. Math., 1967; 15(1).

[17] Kong Fanzhong, Yao Zhenhan, Zheng Xiaoping. BEM for simulation of a 2D elastic body with randomly distributed circular inclusions. Acta Mechanica Solida Sinica, 2002; 15(1): 81 88.

[18] Yao, Zhenhan, Kong, Fanzhong, Wang Pengbo. Simulation of 2D elastic solids with randomly distributed inclusions by boundary element method, in Proc. of WCCM V. Vienna: 2002.

[19] Yao Zhenhan, Wang Pengbo, Kong Fanzhong. Simulation of 2D elastic solids with randomly distributed inclusions without or with interphases by BEM. In Proc. of the $3^{\text {rd }}$ Int. Conf. on Boundary Techniques. Beijing: 2002.

[20] Wang Haitao, Yao Zhenhan. Application of fast multipole BEM for simulation of 2D elastic body with large number of inclusions. In Proc. of the $3^{\text {rd }}$ Int. Conf. on Boundary Techniques. Beijing: 2002. 